\title{
Enriching Medication Review with a Pharmacogenetic Profile - A Case of Tamoxifen Adverse Drug Reactions
}

This article was published in the following Dove Press journal: Pharmacogenomics and Personalized Medicine

\author{
Chiara Jeiziner $\mathbb{D}^{\prime}$ \\ Céline K Stäuble ${ }^{1,2}$ \\ Markus L Lampert ${ }^{\prime}$ \\ Kurt E Hersberger $\mathbb{D}^{\prime}$ \\ Henriette E \\ Meyer zu Schwabedissen ${ }^{2}$ \\ 'Pharmaceutical Care Research Group, \\ Department of Pharmaceutical Sciences, \\ University of Basel, Basel, Switzerland; \\ ${ }^{2}$ Biopharmacy, Department of \\ Pharmaceutical Sciences, University of \\ Basel, Basel, Switzerland
}

\begin{abstract}
Pharmacogenotyping is applied to determine the hereditable component of a patient's susceptibility to experience therapy failure and/or adverse drug reactions (ADRs). We present the case of a female patient diagnosed with breast cancer and treated with tamoxifen as recurrence therapy who experienced various ADRs. Pharmacogenotyping revealed variants in the cytochrome P450 (CYP) enzymes CYP2D6, CYP2C9, and CYP2C19. The observed genotype was associated with a risk for lower tamoxifen efficacy. Aside from the tamoxifen therapy, the comedication was reviewed for the influence of the patient's pharmacogenetic profile. As a result of this pharmacist-led medication review with pharmacogenetic analyses, concrete genotype-driven recommendations for the treating gynecologist were compiled. This case revealed the added value of a large pharmacogenetic panel and the complexity of integrating a pharmacogenetic profile into a recommendation.
\end{abstract}

Keywords: pharmacogenetics, PGx, CYP2D6, CYP2C9, CYP2C19, medication review, tamoxifen

\section{Background}

The first thing a pharmacist does, when a patient reports adverse drug reactions (ADRs), is to go through the patient's medication in order to check for overdosing, contraindications, potential drug-drug or drug-disease interactions, and/ or adherence problems. At the point, where there is no plausible explanation for the reported ADRs, pharmacogenetics (PGx) might help. PGx is the study of genetic variations related to drug response. ${ }^{1}$ Indeed, PGx testing can be applied to determine the hereditable component of a patient's susceptibility to experience therapy failure and/or ADRs. PGx testing aims to identify patients who benefit from a particular drug (responders) or to identify those patients, who carry a predictable risk of non-response or ADRs due to their genetic make-up. The result of pharmacogenotyping has to be evaluated in the context of the active substances taken, therefore also referred to as "stratified pharmacotherapy". 2 It has been shown that pharmacists in the primary care setting are able to contribute to the optimization of the pharmacotherapy by considering the patient's genetic background. ${ }^{3-5}$ One drug that has been extensively studied for the relevance of the patient's genetic predisposition is tamoxifen (TAM). This selective estrogen receptor modulator is used in the prevention and treatment of pre- and postmenopausal breast cancer patients. The endocrine therapy is administered to women
Correspondence: Chiara Jeiziner Pharmaceutical Care Research Group; University of Basel, Petersplatz 14, Postfach 2I48, Basel, 400I, Switzerland Tel +4I 6I 2076180

Email chiara.jeiziner@unibas.ch

Pharmacogenomics and Personalized Medicine 2021:14 279-286 
with estrogen and/or progesterone receptor-positive breast cancer after chemotherapy, radiotherapy, or surgery for 5 years in a dose of $20 \mathrm{mg}$ per day. ${ }^{6}$ By intake of TAM, the annual cancer recurrence rate is halved and the mortality rate is reduced by one-third. ${ }^{7}$

TAM is a prodrug and known for its bioactivation involving multiple cytochrome P450 (CYP) enzymes. In order to form the most active metabolite endoxifen (END), TAM is metabolized either to the intermediate metabolite N-desmethyl-tamoxifen (DM-TAM) or to 4-hydroxytamoxifen (4-OH-TAM). The major metabolite DM-TAM is formed by demethylation catalyzed by CYP3A4 among other CYPs. ${ }^{8}$ DM-TMA is then oxidized by CYP2D6 to END (up to 100 fold more active than TAM) and several other metabolites. Of the remaining TAM, 7\% are oxidized to 4-OH-TAM by CYP2D6 among other CYPs (CYP2C9, CYP2C19 and CYP2B6). ${ }^{8,9}$ In a second step, 4-OH-TAM will be demethylated to END by CYP3A4.

Together with TAM, the three metabolites DM-TAM, 4-OH-TAM, and END can enter the target cancer cells to exert the modulatory effect on estrogen receptors. ${ }^{9}$ Finally, glucuronidation mainly inactivates TAM and its metabolites, so that $75 \%$ of the initial dose will be biliarly excreted as glucuronides. ${ }^{10}$

Regardless of the pathway by which TAM is metabolized, the highly polymorphic CYP2D6 is always involved. Therefore, it is not surprising that changes in CYP2D6 activity associated with inhibition or genetic variants are influencing the bioactivation of TAM. ${ }^{11}$ However, only little is known about the influence of the variability of other CYPs involved in the metabolism of TAM.

\section{Case Presentation}

A premenopausal 49-year-old woman was diagnosed with recurrent breast cancer 12 years after the first diagnosis, where she was initially treated with tumorectomy and sentinel lymphadenectomy, adjuvant radiotherapy, and subsequent endocrine therapy with GnRH-analogs and TAM $20 \mathrm{mg}$ for 24 months. The adjuvant therapy with the GnRHanalogs and TAM was suspended after 2 years, due to various ADRs such as hot flashes, enormous perspirations, psychological distress and the inability to work. Some of these ADRs are known to be linked to TAM therapy. ${ }^{12}$ After the second breast cancer recurrence, a skin-sparing mastectomy with breast reconstruction was performed. Since the initial diagnosis of breast cancer, the treating physician had changed and the new physician was not aware of the previously reported ADRs and therefore, the adjuvant therapy with TAM $20 \mathrm{mg}$ was started again. Due to pronounced climacteric (perspirations and hot flashes) as well as psychological symptoms (irritability, weariness, and depressiveness), the patient asked for a decrease of the TAM dose. Twenty-four months after the re-start of TAM, the dose was decreased to $10 \mathrm{mg}$ per day. Three months later, the patient contacted her gynecologist because ADRs remained and she wanted to learn more about her own TAM response. The gynecologist decided to refer the patient to pharmacists with specific expertise in PGx. The pharmacist performed a medication review supplemented with a pharmacogenetic panel testing. Besides TAM, the patient was taking additional medication (see Table 1 for details). Therefore, the pharmacist also screened comedication for potentially relevant polymorphisms and evaluated potential alternative medication.

\section{Genetic Analyses of Single-Nucleotide Polymorphisms}

After initial consultation and informed consent by the patient following the protocol as approved by the local ethics committee (EKNZ-2019-01452), pharmacogenetic panel testing was conducted applying the commercial test Stratipharm ${ }^{\circledR}$ by humatrix AG (Pfungstadt, Germany), which provides not only the results of genetic testing but also drug-specific interpretation of the corresponding phenotype (pharmacogenetic profile). Table 2 presents the interpretation of a selection of the patient's genotyping results relevant to the herein reported case. The patient held the star alleles *6 (no function) and *41 (decreased function) of the CYP2D6 enzyme resulting in

Table I Patient's Medication at the Time of the Medication Review

\begin{tabular}{|l|l|l|}
\hline Substance & Dosage & Indication \\
\hline $\begin{array}{l}\text { Tamoxifen } \\
10 \mathrm{mg}\end{array}$ & $0-0-\mathrm{I}$ & $\begin{array}{l}\text { Adjuvant endocrine therapy of } \\
\text { breast cancer after mastectomy }\end{array}$ \\
\hline $\begin{array}{l}\text { Mistletoe } \\
\text { preparation }\end{array}$ & As needed & Supportive herbal cancer therapy \\
\hline $\begin{array}{l}\text { Ibuprofen } \\
600 \mathrm{mg}\end{array}$ & $\begin{array}{l}\text { As needed: } \\
0.5-0.5-0.5\end{array}$ & Pain \\
\hline $\begin{array}{l}\text { Metamizole } \\
500 \mathrm{mg}\end{array}$ & As needed & Pain \\
\hline $\begin{array}{l}\text { Pantoprazole } \\
20 \mathrm{mg}\end{array}$ & $\mathrm{I-0-0}$ & As long as therapy with ibuprofen \\
\hline $\begin{array}{l}\text { Lorazepam } \\
\mathrm{mg}\end{array}$ & As needed & Difficulties falling asleep \\
\hline
\end{tabular}


Table 2 Genetic Profile of the Patient

\begin{tabular}{|l|l|l|l|}
\hline Gene & CYP2D6 & CYP2C19 & CYP2C9 \\
\hline $\begin{array}{l}\text { Annotation, } \\
\text { genotype }\end{array}$ & $\begin{array}{l}\text { rs5030655, T/-; } \\
\text { rs2837I725 G/A }\end{array}$ & $\begin{array}{l}\text { rs I2248560 } \\
\text { C/T }\end{array}$ & $\begin{array}{l}\text { rs I799853 } \\
\text { C/T }\end{array}$ \\
\hline Haplotypes & $* 6 / * 4 I$ & $* I / * 17$ & $* I / * 2$ \\
\hline $\begin{array}{l}\text { Predicted } \\
\text { phenotype }\end{array}$ & IM & UM & IM \\
\hline
\end{tabular}

Abbreviations: IM, intermediate metabolizer; UM, ultrarapid metabolizer.

the phenotype of intermediate metabolizer (IM). At the same time, the patient had a CYP2C19 ultrarapid metabolizer (UM) status with the star alleles *1 (wildtype) and *17 (increased function) and a CYP2C9 IM status with the star alleles *1 (wildtype) and $* 2$ (decreased function).

For TAM, Stratipharm ${ }^{\circledR}$ reported the CYP2D6 IM status, which is linked to reduced enzyme activity, and consequently, an insufficient activation of the prodrug TAM to the major active metabolite END. Stratipharm ${ }^{\circledR}$ did not report results related to any other genes relevant to the TAM metabolism. For pantoprazole, the increased enzyme activity of CYP2C19 may lead to increased degradation of the metabolized substance. Therefore, ineffectiveness of pantoprazole was predicted for standard dosage in our patient. For ibuprofen, the decreased enzyme activity of CYP2C9 may lead to an accumulation of the metabolites. Consequently, the patient has a higher risk of ADRs, eg gastrointestinal bleedings.

\section{Discussion and Decision-Making}

Both genetic predisposition and the patient's medication were considered when evaluating the reported ADRs observed during TAM treatment. We will start with the description of our considerations for TAM, as we assumed the ADRs to be linked to the treatment with the selective estrogen receptor modulator, even though we are aware that the pathophysiology of hot flushes especially in breast cancer survivors is not fully understood. ${ }^{13}$ Based on our knowledge on TAM metabolism and its function as selective estrogen receptor modulator, we expected a CYP2D6 UM status leading to an excess of END, thereby resulting in the reported ADRs. However, the pharmacogenetic profile showed that the patient had the star alleles $* 6$ (no function) and $* 41$ (decreased function) of the CYP2D6 enzyme resulting in the phenotype of an IM. Moreover, the testing revealed the patient's CYP2C19 UM status with the star alleles *1 (wildtype) and *17 (increased function), and a CYP2C9 IM status with the star alleles *1 (wildtype) and *2 (decreased function).

If PGx data for a patient are available, one should be able to consult the official drug label or the guidelines by the respective medical expert panel for information on the handling of a drug. For TAM, Swiss drug labels (www. swissmedicinfo.ch) does not recommend CYP2D6 testing, but simply states, that in published studies, the simultaneous use of CYP2D6 inhibitors reduced plasma concentrations of the active metabolite endoxifen, which may be associated with a loss of efficacy (see 'Interactions'). According to a published study, the loss of efficacy of TAM in combination with treatment with SSRI paroxetine increased mortality. TAM should therefore not be administered together with CYP2D6 inhibitors (eg SSRI antidepressants such as paroxetine or fluoxetine, cinacalcet, quinidine). Reduced endoxifen concentrations and thus reduced efficacy can also be expected in so-called poor metabolizers for CYP2D6 (see 'Pharmacokinetics' and 'Properties/Effects'). ${ }^{14}$ This information is similar to the drug label issued by the FDA and Health Canada. $^{15}$ Finally, the National Comprehensive Cancer Network (NCCN) Breast Cancer Panel does not recommend CYP2D6 genotyping. ${ }^{16}$ Accordingly, these sources did not provide the information we were looking for.

The Clinical Pharmacogenetics Implementation Consortium (CPIC) or the Dutch Pharmacogenetics Working Group (DWPG) provide recommendations on the implementation of PGx information in medical decisions for a selection of drugs including TAM. In their expert summary on TAM, both report a clear association of the herein observed genotype of CYP2D6 *6/*41 (IM), with lower END levels and with a higher risk of breast cancer recurrence in premenopausal women. In the case of a CYP2D6 IM status, they recommend considering alternative substances for the endocrine therapy such as aromatase inhibitors (AIs). ${ }^{17-19}$ In their update of guidelines, Swen et $\mathrm{al}^{19}$ confirmed an increased recurrence rate of breast cancer and strongly recommended to avoid the use of potent CYP2D6 inhibitors for CYP2D6 IMs. For postmenopausal women, they suggested considering the use of AIs, even though two large randomized double-blind trials $^{20,21}$ concluded that the CYP2D6 genotype does not predict the clinical outcome with TAM. However, there are studies confirming ${ }^{22-24}$ and studies contradicting ${ }^{20,21}$ the predictive value of the CYP2D6 genotype for the clinical outcome, which certainly contributes to the lack of agreement on the pharmacogenotyping prior to TAM treatment. 
There are data suggesting that despite the enhanced activation of TAM to END in patients with UM status, this status is linked to a higher intake of symptom-relieving drugs (antinausea, anxiolytics, medications for relief from hot flushes), a higher frequency of early treatment discontinuation, and a worse prognosis for breast cancer compared to extensive metabolizers. ${ }^{25}$

Another option to overcome the decreased END levels in patients with CYP2D6 IM status might be a doseescalation, but this was not recommended in the guidelines of the CPIC/DWPG and no option for our patient, suffering from various ADRs from TAM. Nevertheless, we would like to mention that there are experts ${ }^{26}$ reporting that there is no impact on quality of life when applying monitoring of END plasma concentrations and according to TAM dose escalation in CYP2D6 poor metabolizers (PMs). Besides, we were unable to collect blood samples in the community pharmacy.

Toremifene is another selective estrogen receptor modulator indicated for the treatment of metastatic breast cancer in postmenopausal women. ${ }^{27}$ Even if CYP2D6 is involved in the toremifene metabolism as shown in vitro, ${ }^{28}$ this enzyme seems to play a minor role in vivo. ${ }^{29}$ Within the herein reported case, toremifene was no therapeutic option as the drug has been discontinued in the Swiss market.

As it remained unclear whether the observed ADRs are linked to the patient's genotype, we focused on the bioactivation of TAM. The first step, namely the demethylation of TAM to DM-TAM, which is considered as the main pathway of TAM degradation, ${ }^{10}$ is mainly catalyzed by CYP3A4. ${ }^{930}$ However, for CYP3A4 no function predicting polymorphisms exist. ${ }^{31}$ Furthermore, it is well known that CYP2D6 is a major contributor to the hydroxylation both of TAM to the intermediate metabolite 4-OH-TAM and of the intermediate metabolite DM-TAM to END. If less CYP2D6 is available, we must assume both steps to be slower. Consequently, smaller amounts of END might be formed. In 2009, Schroth et $\mathrm{al}^{22}$ retrospectively genotyped a cohort of 1325 patients with breast cancer at an early stage and demonstrated that the existence of a nonfunctional (PM) or reduced-function allele (IM) instead of two functional alleles of CYP2D6 lead to worse clinical outcomes. Even though there is a link between the bioactivation of TAM and CYP2C19, the DPWG and CPIC guidelines do not consider this relationship. Indeed, CYP2C19 is known to be capable of catalyzing the 4-hydroxylation to 4-OH-TAM and the demethylation to
END. ${ }^{11}$ In addition, there are data linking CYP2C19 to both estrogen and progesterone metabolism. ${ }^{32}$ In detail, the authors demonstrated in a cohort study with 306 preand post-menopausal women, that patients with a decreased activity of the CYP2D6 enzyme, thus poor activation of TAM, in combination with an increased activity in CYP2C19 UM, thus lower levels of sexual hormones, have worse clinical outcome. Looking only at CYP2C19, it has been shown that the CYP2C19*17 allele (UM) is associated with a better response to the TAM therapy, fewer ADRs, and disease-free survival. ${ }^{10,33}$ Furthermore, Schroth et al report an analysis considering both the CYP2D6 and the CYP2C19*17 genotype revealing a more accurate stratification of the patients with poor and moderate outcomes when also considering CYP2C19*17. ${ }^{33}$ In addition, the CYP2C9 is assumed to be involved in the formation of the intermediate metabolites DM-TAM and 4-OH-TAM. ${ }^{11}$ Therefore, the CYP2C9 IM status of our patient might have led to a decreased level of the intermediate metabolites DM-TAM and 4-OHTAM. Finally, CYP3A4 is involved in the demethylation of TAM to DM-TAM, and of 4-OH-TAM to END. ${ }^{9,10,30}$ However, based on the current understanding genetic variants in CYP3A4 are not predictive for its metabolic activity, but CYP3A4 is often involved in drug-drug interactions either increasing or decreasing its activity. Taken together, the genetic findings suggest that the herein reported patient is carrying a risk for lower TAM efficacy (CYP2D6 IM, CYP2C9 IM), which is slightly reduced by the enhanced activity of CYP2C19.

Even though an initial screening of drug-drug interactions using the interaction tool MediQ (www.mediq.ch) did not show any significant drug-drug interactions, we performed an in-depth analysis of the potential drug-drug interactions. On one hand, there is the possibility of induction of enzymes involved in TAM metabolism. This is especially known for CYP3A4, but also for other enzymes involved in TAM metabolism such as CYP2C9 and CYP2C19. ${ }^{31}$ However, there are no drugs present in the patient's comedication currently known to induce CYP3A4, CYP2C9, and CYP2C19 function. On the other hand, processes of inhibition are possible. In the context of TAM comedication, it seems noteworthy that pantoprazole is considered to be a CYP2C19 substrate, and therefore might have had a weak inhibitory effect on the increased activity of CYP2C19 in the patient. ${ }^{34}$ Another compound, that may influence drug metabolism is the herbal medication Viscum album taken by the patient 
as a complementary therapy. Weissenstein et $\mathrm{al}^{35}$ treated the human breast cancer cell line MCF-7 with END, either with or without the presence of estradiol. Viscum album extracts were added to all samples and proliferation, apoptosis and cell cycle were analyzed. In addition, possible inhibition of CYP3A4/5 and CYP2D6 was examined in human liver microsomes. Viscum album did neither influence the anti-estrogenic effect of endoxifen; nor interact with the bioactivation of TAM.

After consulting drug labels, guidelines, and literature, it still remained unclear whether the observed ADRs are linked to the patient's genotype. In this context, we want to cite the results from Regan et al ${ }^{21}$ investigating the development of hot flashes in 1706 patients treated with TAM in the first 2 years of treatment. Contrary to the hypothesis of worse disease control in patients with CYP2D6 PM and IM phenotypes, they found an increased risk for development of hot flashes in patients with CYP2D6 PM and IM phenotypes. No such association was observed by Sestak et $\mathrm{al}^{36}$ assessing the same outcome in a smaller patient population $(\mathrm{n}=54)$. According to Vries et $\mathrm{al}^{11}$, PGx alone cannot answer the question of the reactions on treatment with TAM, as this depends on multiple factors, eg the menopausal status of the woman. For the herein presented case, we are unable to explain the ADRs, but the CYP2D6 IM status of the patient let us to decide that TAM is not a good treatment option. Testing the patient for the END plasma levels in a therapeutic drug monitoring would have certainly helped to provide further insights into the case. ${ }^{37}$ However, this is not applicable in the herein presented setting of patient care in a community pharmacy.

Finally, we want to refer to the single drug analysis of the patient's comedication (see Table 1), which was possible due to the pharmacogenetic profile, and which influenced the pharmacist's recommendations for this particular patient. The first active substance we want to mention is pantoprazole. A few studies have demonstrated that CYP2C19 is an important determinant in its metabolism ${ }^{38-40}$ linking changes in plasma levels to the phenotype of CYP2C19 metabolism. ${ }^{38}$ The recommendations on PGx implementation suggest a fourfold $(400 \%)$ elevation in the dose of pantoprazole in the therapy of the $H$. pylori infection and other indications to ensure the intended pharmacological activity. ${ }^{19}$ The majority of drugs in the class of proton pump inhibitors are mainly metabolized via CYP2C19. However, rabeprazole is metabolized via a non-enzymatic pathway; therefore, it is less susceptible to the influence of genetic polymorphisms of CYP2C19. ${ }^{41}$
The second active substance in this context is ibuprofen, which is metabolized by CYP2C9, CYP2C8, CYP2C19 and multiple UDP-glucuronosyltransferases, where CYP2C9 is assumed to be of major relevance. ${ }^{42}$ In 2020, the CPIC published a guideline for non-steroidal anti-inflammatory drugs (NSAIDs) in the context of genetic variants of $\mathrm{CYP} 2 \mathrm{C} 8$ and $\mathrm{CYP} 2 \mathrm{C}^{43}$, where IMs might have a higher risk for adverse events such as gastrointestinal bleeding than others. They recommend starting the therapy with the lowest effective dose for the shortest duration and in agreement with patients' preferences.

\section{Decision}

We recommended to discontinue TAM due to the CYP2D6 IM phenotype and the fact that dose escalation would not be an option for this patient due to ADRs, which might be linked to the genetic-associated changes in TAM metabolism. In the recommendation, we suggested a switch to letrozole, a non-steroidal AI for post-breast cancer recurrence therapy after breast cancer. AIs represent a good alternative to TAM also supported by a meta-analysis of the Early Breast Cancer Trials Collaborative Group. Here, the comparison of TAM and AIs revealed that the recurrence rates of AIs are reduced by $30 \%$ compared to TAM and that 10 -year mortality is reduced by $15 \%$ by AIs compared to TAM. ${ }^{44}$ Jeong et $\mathrm{al}^{45}$ also support the switch to AIs as they have shown to be superior to TAM. ${ }^{16}$ Furthermore, letrozole is primarily catalyzed by CYP2A6 and CYP3A4 and it is assumed to be independent of metabolism by CYP2D6, CYP2C9 and CYP2C19.

In addition, we recommended ibuprofen for short-term intake only and advised the additional intake of a proton pump inhibitor, as the CYP2C9 IM status may increase the risk of gastrointestinal bleedings during the ibuprofen therapy. For the proton pump inhibition, we recommended discontinuing pantoprazole, due to the CYP2C19 UM status which might lead to subtherapeutic pantoprazole plasma levels and suggest to switch to rabeprazole, which is mainly metabolized by a non-enzymatic pathway.

\section{Follow-Up}

The recommendations were forwarded to the treating gynecologist, who decided - together with the patient to start letrozole in combination with the GnRH-agonist leuprorelin (also known as leuprolide). Despite the patient's fear of further ADRs caused by the new treatment, the pharmacogenetic profile provided sufficient argumentation for the patient to adapt her post-breast 
cancer recurrence therapy. Accordingly, TAM was stopped and switched to a monthly injection of leuprorelin $2.75 \mathrm{mg}$ (due to the premenopausal status of the patient) and supplemented by letrozole $2.5 \mathrm{mg}$ once daily initiated 2 weeks after the initiation of leuprorelin. Six months after the initiation of letrozole and leuprorelin, the patient reported a minimal local reaction at the injection site of the leuprorelin, but she does not suffer from any other ADRs. Moreover, the patient was also able to go back to work part-time. Furthermore, the patient stopped the ibuprofen treatment, and therefore the proton pump inhibitor was not indicated anymore to prevent gastrointestinal bleeding, but the patient wanted to have rabeprazole on-hold in case of an ibuprofen intake in the future.

\section{Conclusion}

This case presents a patient showing ADRs in the adjuvant therapy with TAM. We analyzed the patient's medication and used a pharmacogenetic profile provided by a commercially available panel comprising 30 genes with up to 100 variants and drug-specific interpretation. Relevant variants in the CYP enzymes CYP2D6, CYP2C9, and CYP2C19 were detected. As a result of this pharmacist-led medication review with pharmacogenetic analyses, concrete genotype-driven recommendations for the treating physician were compiled.

At first, considering only the described ADRs would have tempted us to reduce the TAM dosage, assuming that the patient's END blood concentrations might be elevated. Surprisingly, the assessed CYP2D6 genotype (CYP2D6 IM) did not support this hypothesis. On the contrary, respecting the PGx guidelines ${ }^{16,18,19}$ according to the revealed CYP2D6 genotype, the patient might suffer from limited TAM effectiveness due to decreased bioactivation to END via CYP2D6 and therefore will not benefit from a dose reduction. Consequently, we recommended to stop TAM and switch to letrozole in combination with leuprorelin. Furthermore, we were able to deliver recommendations to the comedication of the patient.

This case showed the added value of a large pharmacogenetic panel and the complexity of integrating a pharmacogenetic profile into a recommendation. Consequently, the decision-making process needs an interdisciplinary approach for the clinical implementation of PGx.

\section{Acknowledgments}

We would like to thank Tamara Imfeld-Isenegger for proofreading the manuscript.

\section{Author Contributions}

Chiara Jeiziner led the execution of the report and was part of conception, study design, acquisition of data, analysis and interpretation of the case. She drafted the article. Céline Stäuble was part of analysis and interpretation of the case. She reviewed the article critically. Markus Lampert was part of conception and interpretation of the case. He reviewed the article critically. Kurt Hersberger was part of conception, study design, execution, and interpretation of the case. He reviewed the article critically. Henriette Meyer zu Schwabedissen was part of conception and study design of the case and she led data analysis and interpretation. She revised the article. All the authors have agreed on the submission of the article to the journal Pharmacogenomics and Personalized Medicine. All the authors reviewed and agreed on all versions of the article before submission, and during revision, accepted the final version for publication, and any significant changes introduced at the proofing stage. All the authors agree to take responsibility and be accountable for the contents of the article.

\section{Ethics Disclosure}

The patient has provided informed consent for the use of her data as well as for the publishing of the case details for research purposes. The case was collected in the framework of the observational study "Pharmacogenetic Testing of Patients with unwanted Adverse Drug Reactions or Therapy Failure" approved by the local ethics committee (PGx-exHerberger-01452) on 31.10.2019.

\section{Disclosure}

There are no conflicts of interest.

\section{References}

1. ICH Topic E15. Definitions of genomic biomarkers, pharmacogenomics, pharmacogenetics, genomic data, sample coding categories. 2007. Available from: https://www.ema.europa.eu/en/documents/scientificguideline/ich-e-15-definitions-genomic-biomarkers-pharmacogenomicspharmacogenetics-genomic-data-sample-coding_en.pdf. Accessed July 10, 2019.

2. Meyer $\mathrm{Zu}$ Schwabedissen HE. The Role of Pharmacogenomics in Individualized Medicine. Springer; 2015:93-112.

3. Dunnenberger HM, Crews KR, Hoffman JM, et al. Preemptive clinical pharmacogenetics implementation: current programs in five US medical centers. Annu Rev Pharmacol Toxicol. 2015;55:89-106. doi:10.1146/annurev-pharmtox-010814-124835

4. Roden DM, Van Driest SL, Mosley JD, et al. Benefit of preemptive pharmacogenetic information on clinical outcome. Clin Pharmacol Ther. 2018;103:787-794. doi:10.1002/cpt.1035

5. Bank PCD, Swen JJ, Guchelaar HJ. Estimated nationwide impact of implementing a preemptive pharmacogenetic panel approach to guide drug prescribing in primary care in The Netherlands. BMC Med. 2019;17:110. doi:10.1186/s12916-019-1342-5 
6. S3-Leitlinie Mammakarzinom Version 4.2; 2020. Available from: https://www.leitlinienprogramm-onkologie.de/fileadmin/user_upload/ Downloads/Leitlinien/Mammakarzinom_4_0/Version_4.3/LL_ Mammakarzinom_Kurzversion_4.2.pdf. Accessed July 15, 2020.

7. Early Breast Cancer Trialists' Collaborative Group. Relevance of breast cancer hormone receptors and other factors to the efficacy of adjuvant tamoxifen: patient-level meta-analysis of randomised trials. Lancet. 2011;378:771-784. doi:10.1016/S0140-6736(11)60993-8

8. Mürdter TE, Schroth W, Bacchus-Gerybadze L, et al. Activity levels of tamoxifen metabolites at the estrogen receptor and the impact of genetic polymorphisms of Phase I and II enzymes on their concentration levels in plasma. Clin Pharmacol Ther. 2011;89:708-717. doi:10.1038/clpt.2011.27

9. Desta Z, Ward BA, Soukhova NV, Flockhart DA. Comprehensive evaluation of tamoxifen sequential biotransformation by the human cytochrome P450 system in vitro: prominent roles for CYP3A and CYP2D6. J Pharmacol Exp Therap. 2004;310:1062-1075. doi:10.1124/jpet.104.065607

10. Klein DJ, Thorn CF, Desta Z, Flockhart DA, Altman RB, Klein TE. PharmGKB summary: tamoxifen pathway, pharmacokinetics. Pharmacogenet Genomics. 2013;23:643-647. doi:10.1097/ FPC.0b013e3283656bc1

11. de Vries Schultink AHM, Zwart W, Linn SC, Beijnen JH, Huitema ADR. Effects of pharmacogenetics on the pharmacokinetics and pharmacodynamics of tamoxifen. Clin Pharmacokinet. 2015;54:797-810. doi:10.1007/s40262-015-0273-3

12. Moon Z, Hunter MS, Moss-Morris R, Hughes LD. Factors related to the experience of menopausal symptoms in women prescribed tamoxifen. J Psychosom Obstet Gynaecol. 2017;38:226-235. doi:10.1080/0167482X.2016.1216963

13. Lee CI, Fox P, Balakrishnar B, et al. Tamoxifen-induced severe hot flashes and endoxifen levels: is dose reduction a safe and effective strategy? Breast. 2019;46:52-57. doi:10.1016/j.breast.2019.05.009

14. Jeiziner C, Suter K, Wernli U, et al. Pharmacogenetic information in Swiss drug labels - a systematic analysis. Pharmcogenom J. 2020.

15. Tamoxifen pathway; 2020. Available from: https://www.pharmgkb. org/pathway/PA145011119. Accessed June 12, 2020.

16. Dean L. Tamoxifen Therapy and CYP2D6 Genotype. Medical Genetics Summaries [Internet]: National Center for Biotechnology Information (US); 2019.

17. Pharmacogenetics -recommendations and background information enzymes; 2020. Available from: https:/www.knmp.nl/patientenzorg/ medicatiebewaking/farmacogenetica/pharmacogenetics-1/pharmaco genetics. Accessed February 01, 2020.

18. Goetz MP, Sangkuhl K, Guchelaar HJ, et al. Clinical Pharmacogenetics Implementation Consortium (CPIC) guideline for CYP2D6 and tamoxifen therapy. Clin Pharmacol Ther. 2018;103:770-777. doi:10.1002/cpt.1007

19. Swen JJ, Nijenhuis M, de Boer A, et al. Pharmacogenetics: from bench to byte-an update of guidelines. Clin Pharmacol Ther. 2011;89:662-673. doi:10.1038/clpt.2011.34

20. Rae JM, Drury S, Hayes DF, et al. CYP2D6 and UGT2B7 genotype and risk of recurrence in tamoxifen-treated breast cancer patients. J Natl Cancer Inst. 2012;104:452-460. doi:10.1093/jnci/djs126

21. Regan MM, Leyland-Jones B, Bouzyk M, et al. CYP2D6 genotype and tamoxifen response in postmenopausal women with endocrine-responsive breast cancer: the breast international group 1-98 trial. J Natl Cancer Inst. 2012;104:441-451. doi:10.1093/jnci/djs125

22. Schroth W, Goetz MP, Hamann U, et al. Association between CYP2D6 polymorphisms and outcomes among women with early stage breast cancer treated with tamoxifen. JAMA. 2009;302:1429-1436. doi:10.1001/jama.2009.1420

23. Kiyotani K, Mushiroda T, Imamura CK, et al. Significant effect of polymorphisms in $\mathrm{CYP} 2 \mathrm{D} 6$ and $\mathrm{ABCC} 2$ on clinical outcomes of adjuvant tamoxifen therapy for breast cancer patients. J Clin Oncol. 2010;28:1287-1293. doi:10.1200/JCO.2009.25.7246
24. Goetz MP, Suman VJ, Hoskin TL, et al. CYP2D6 metabolism and patient outcome in the Austrian Breast and Colorectal Cancer Study Group trial (ABCSG) 8. Clin Cancer Res. 2013;19:500-507. doi:10.1158/1078-0432.CCR-12-2153

25. He W, Grassmann F, Eriksson M, et al. CYP2D6 genotype predicts tamoxifen discontinuation and prognosis in patients with breast cancer. J Clin Oncol. 2020;38:548-557. doi:10.1200/JCO.19.01535

26. Hertz DL, Deal A, Ibrahim JG, et al. Tamoxifen dose escalation in patients with diminished CYP2D6 activity normalizes endoxifen concentrations without increasing toxicity. Oncologist. 2016;21:795-803. doi:10.1634/theoncologist.2015-0480

27. Vogel CL, Johnston MA, Capers C, Braccia D. Toremifene for breast cancer: a review of 20 years of data. Clin Breast Cancer. 2014;14:1-9. doi:10.1016/j.clbc.2013.10.014

28. Watanabe M, Watanabe N, Maruyama S, Kawashiro T. Comparative metabolic study between two selective estrogen receptor modulators, toremifene and tamoxifen, in human liver microsomes. Drug Metab Pharmacokinet. 2015;30:325-333. doi:10.1016/j.dmpk.2015.05.004

29. Kim J, Dalton JT, Veverka KA. Relationship of CYP2D6 status and toremifene metabolism. J Clin Oncol. 2011;29:e13068-e. doi:10.1200/jco.2011.29.15_suppl.e13068

30. Crewe HK, Notley LM, Wunsch RM, Lennard MS, Gillam EM. Metabolism of tamoxifen by recombinant human cytochrome P450 enzymes: formation of the 4-hydroxy, 4'-hydroxy and n-desmethyl metabolites and isomerization of trans-4-hydroxytamoxifen. Drug Metab Dispos. 2002;30:869-874. doi:10.1124/dmd.30.8.869

31. Zanger UM, Schwab M. Cytochrome P450 enzymes in drug metabolism: regulation of gene expression, enzyme activities, and impact of genetic variation. Pharmacol Ther. 2013;138:103-141.

32. Sim S, Lövrot J, Lindh JD, Bergh J, Xie H. Effect of CYP2C19 and CYP2D6 genotype on tamoxifen treatment outcome indicates endogenous and exogenous interplay. Pharmacogenomics. 2018;19:1027-1037. doi:10.2217/pgs-2018-0089

33. Schroth W, Antoniadou L, Fritz P, et al. Breast cancer treatment outcome with adjuvant tamoxifen relative to patient CYP2D6 and CYP2C19 genotypes. J Clin Oncol. 2007;25:5187-5193. doi:10.1200/JCO.2007.12.2705

34. Desta Z, Zhao X, Shin JG, Flockhart DA. Clinical significance of the cytochrome P450 2C19 genetic polymorphism. Clin Pharmacokinet. 2002;41:913-958.

35. Weissenstein U, Kunz M, Oufir M, et al. Absence of herb-drug interactions of mistletoe with the tamoxifen metabolite (E/Z)-endoxifen and cytochrome P450 3A4/5 and 2D6 in vitro. BMC Complement Altern Med. 2019;19:23. doi:10.1186/s12906-019-2439-2

36. Sestak I, Kealy R, Nikoloff M, et al. Relationships between CYP2D6 phenotype, breast cancer and hot flushes in women at high risk of breast cancer receiving prophylactic tamoxifen: results from the IBIS-I trial. $\mathrm{Br} J$ Cancer. 2012;107:230-233. doi:10.1038/ bjc. 2012.278

37. de Vries Schultink AHM, Huitema ADR, Beijnen JH. Therapeutic drug monitoring of endoxifen as an alternative for CYP2D6 genotyping in individualizing tamoxifen therapy. Breast. 2018;42:38-40. doi:10.1016/j.breast.2018.08.100

38. Gawronska-Szklarz B, Adamiak-Giera U, Wyska E, et al. CYP2C19 polymorphism affects single-dose pharmacokinetics of oral pantoprazole in healthy volunteers. Eur $J$ Clin Pharmacol. 2012;68:1267-1274. doi:10.1007/s00228-012-1252-3

39. Román M, Ochoa D, Sánchez-Rojas SD, et al. Evaluation of the relationship between polymorphisms in CYP2C19 and the pharmacokinetics of omeprazole, pantoprazole and rabeprazole. Pharmacogenomics. 2014;15:1893-1901.

40. Hunfeld NG, Touw DJ, Mathot RA, et al. A comparison of the acid-inhibitory effects of esomeprazole and pantoprazole in relation to pharmacokinetics and CYP2C19 polymorphism. Aliment Pharmacol Ther. 2010;31:150-159. doi:10.1111/j.1365-2036.2009. 04150.x 
41. Pace F, Pallotta S, Casalini S, Porro GB. A review of rabeprazole in the treatment of acid-related diseases. Ther Clin Risk Manag. 2007;3:363-379.

42. Mazaleuskaya LL, Theken KN, Gong L, et al. PharmGKB summary: ibuprofen pathways. Pharmacogenet Genomics. 2015;25:96-106. doi:10.1097/FPC.0000000000000113

43. Katherine N, Theken CRL, Gong L, et al. CPIC guideline for NSAID and CYP2C9. 2020.

44. Early Breast Cancer Trialists' Collaborative Group. Aromatase inhibitors versus tamoxifen in early breast cancer: patient-level meta-analysis of the randomised trials. Lancet (London, England). 2015;386:1341-1352. doi:10.1016/S0140-6736(15)61074-1
45. Jeong S, Woo MM, Flockhart DA, Desta Z. Inhibition of drug metabolizing cytochrome P450s by the aromatase inhibitor drug letrozole and its major oxidative metabolite 4,4'-methanol-bisbenzonitrile in vitro. Cancer Chemother Pharmacol. 2009;64:867-875. doi:10.1007/s00280-009-0935-7

\section{Publish your work in this journal}

Pharmacogenomics and Personalized Medicine is an international, peer-reviewed, open access journal characterizing the influence of genotype on pharmacology leading to the development of personalized treatment programs and individualized drug selection for improved safety, efficacy and sustainability. This journal is indexed on the American Chemical Society's Chemical Abstracts Service (CAS). The manuscript management system is completely online and includes a very quick and fair peer-review system, which is all easy to use. Visit http://www.dovepress.com/testimonials.php to read real quotes from published authors. 